\title{
SMOKING ASSOCIATED WITH CHRONIC OBSTRUCTIVE PULMONARY DISEASE, ACCELERATED HYPERTENSION, SPONTANEOUS PNEUMOTHORAX, MEDIASTINAL EMPHYSEMA LARGE AORTIC ANEURYSM AND PULMONARY TUBERCULOSIS - A CASE REPORT
}

\author{
Verma P. K. ${ }^{1}$, Rajpal S. ${ }^{2}$, Ranga G. S. ${ }^{3}$, Dwivedi S4 \\ 1 Lecturer \\ 2Senior Medical Specialist \\ 3Professor \\ ${ }^{4}$ Professor and Head Department of Medicine, \\ University College of Medical Sciences, \\ University of Delhi \& GTB Hospital, Delhi -110095 \\ India
}

\begin{abstract}
:
Chronic smoking is often associated with chronic obstructive pulmonary disease, coronary artery disease, hypertension \& aortic aneurysm in elderly people. However its life threatening complications in the form of tension pneumothorax, mediastinal emphysema, and enlarging aortic aneurysm coexisting with pulmonary tuberculosis at times poses diagnostic and therapeutic challenge. We report here with an 86 - year - old male who had mediastinal emphysema, large aortic aneurysm, accelerated hypertension and evidence of active pulmonary tuberculosis aggravated by chronic smoking. He made remarkable recovery following intercostal drainage, anti tuberculous and supportive intensive therapy.
\end{abstract}

Keywords: Chronic obstructive pulmonary disease, Pneumothorax, Mediastinal emphysema, Aortic aneurysm, Hypertension, Pulmonary tuberculosis

\section{Correspondence to:}

Dr. Pushpendra K Verma

B-355/A, Ashok Nagar,

Mandoli Road,

Delhi-110093 (INDIA)

Ph.: 91-9868875677

E-mail: pkumarv2002@gmail.com 


\section{Introduction}

Chronic smoking is associated with chronic obstructive pulmonary disease (COPD), coronary artery disease (CAD), hypertension (HTN) \& aortic aneurysm (AA) \& several types of cancer. There is always a potential danger of rupture of large aortic aneurysm. Besides this the other complication of bullous lesion associated with COPD is spontaneous pneumothorax. We recently had a male octogenerian who presented to us with the spontaneous pneumothorax, extensive subcutaneous emphysema associated with mediastinal emphysema, accelerated hypertension \& active pulmonary tuberculosis. The diagnostic \& therapeutic challenge associated with such unusual

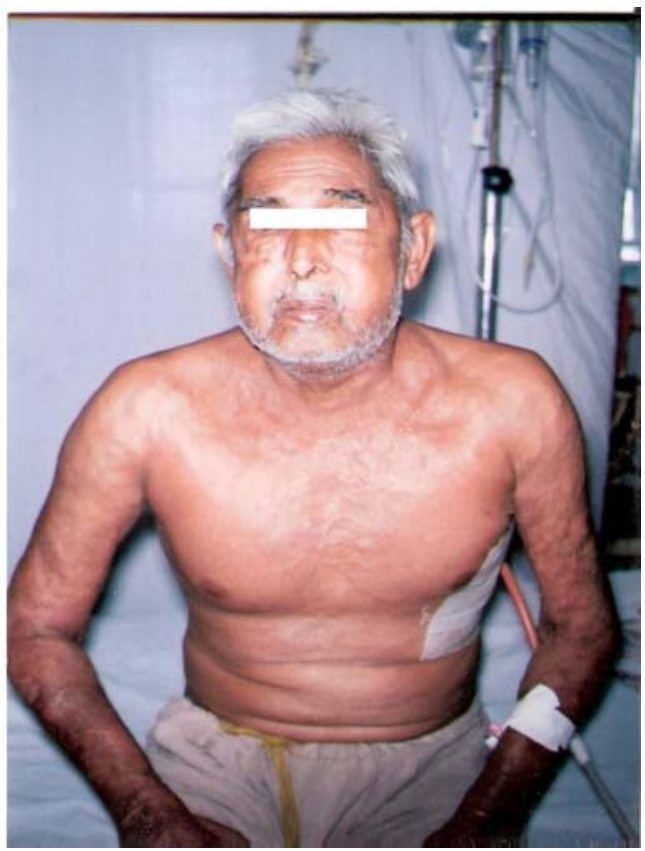

Fig. 1

Showing extensive subcutaneous emphysema extending to face, arms and chest

In past he was diagnosed to have pulmonary tuberculosis twice once in 2003 and then complications of chronic smoking prompted us to report this case.

Case Report: A, 86 - year - old man, presented to medical emergency with acute onset breathlessness which was progressive in nature since 1 month and off and on cough with occasional expectoration since 15 days. Urgent chest skiagram revealed left sided pneumothorax with features of COPD, dilated and tortuous, ascending, arch and descending aorta and aortic calcification. Blood pressure was $220 / 110 \mathrm{mmHg}$. An intercostal tube was inserted with underwater seal. He accidentally removed the intercostal tube and developed extensive subcutaneous emphysema involving lower half of both sides of chest wall, arms, neck, face and periorbital area. (Fig.1). Patient became acutely dyspneic at this stage.

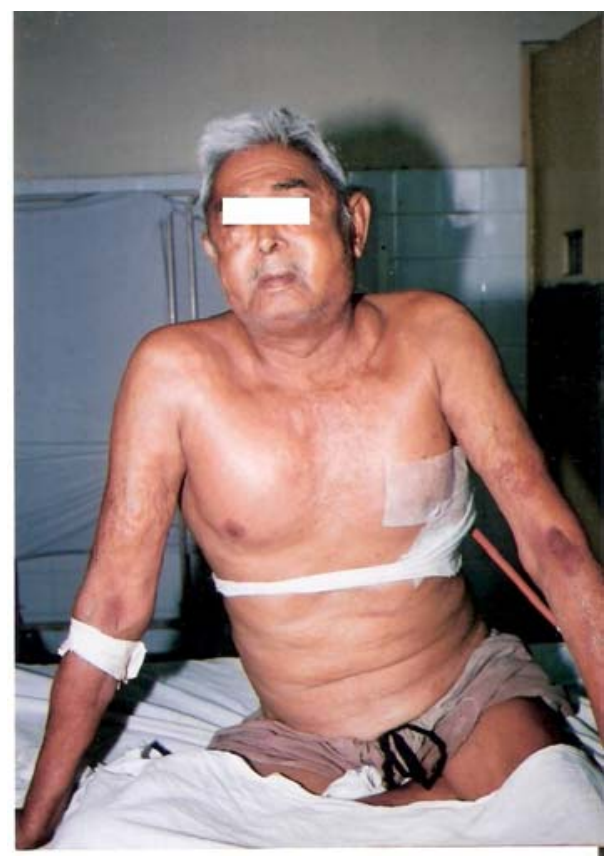

Fig. 2

After regression of subcutaneous emphysema

again in 2005 for which he received anti tubercular treatment albeit for 2 months only 
both times in the form of RHZE. Beside this he had difficulty in micturition for 10 years and was diagnosed to have benign prostatic hyperplasia for which surgery was planned a year back. As pre-anesthetic check up x-ray chest revealed aortic aneurysm, the prostatic surgery was deferred. His family history- was unremarkable. He was a chronic smoker (10 bidis per day for last 50 years), has been smoking till the episode of acute chest pain, he occasionally took alcohol also.

At the time of admission he was conscious, blood pressure-170/110 $\mathrm{mmHg}$ and pulse $84 /$ min. All peripheral pulses were palpable except left dorsalis pedis. There was mild pallor. Trachea was shifted to right side; Movement was diminished on left side. Supraclavicular fosses were full. Hyperresonant tympanic note was heard on left side. Crepitus was felt all over neck, anterior chest wall, back and upper part of abdomen. Breath sounds and vocal resonance were diminished on left side. Based on clinical and radiological finding a presumptive clinical diagnosis of COPD, accelerated hypertension, left pneumothorax, extensive subcutaneous \& mediastinal emphysema, large aortic aneurysm and pulmonary tuberculosis was made. Investigations revealed low HDL (36mg/dl), low voltage ECG complexes and a negative VDRL. Due to all pervasive gas and poor echogenic window Echo and carotid intima media thickness could not be performed.

In view of increasing dyspnea and extensive subcutaneous emphysema and large aortic aneurysm contrast C.T scanning of the chest was done. This revealed extensive subcutaneous emphysema along with mediastinal and retroperitoneal emphysema involving chest and abdominal wall, multiple bilateral bullae, right fibrocavitory lesions at apex, left sided pneumothorax, Fig. 3 \& 4 .
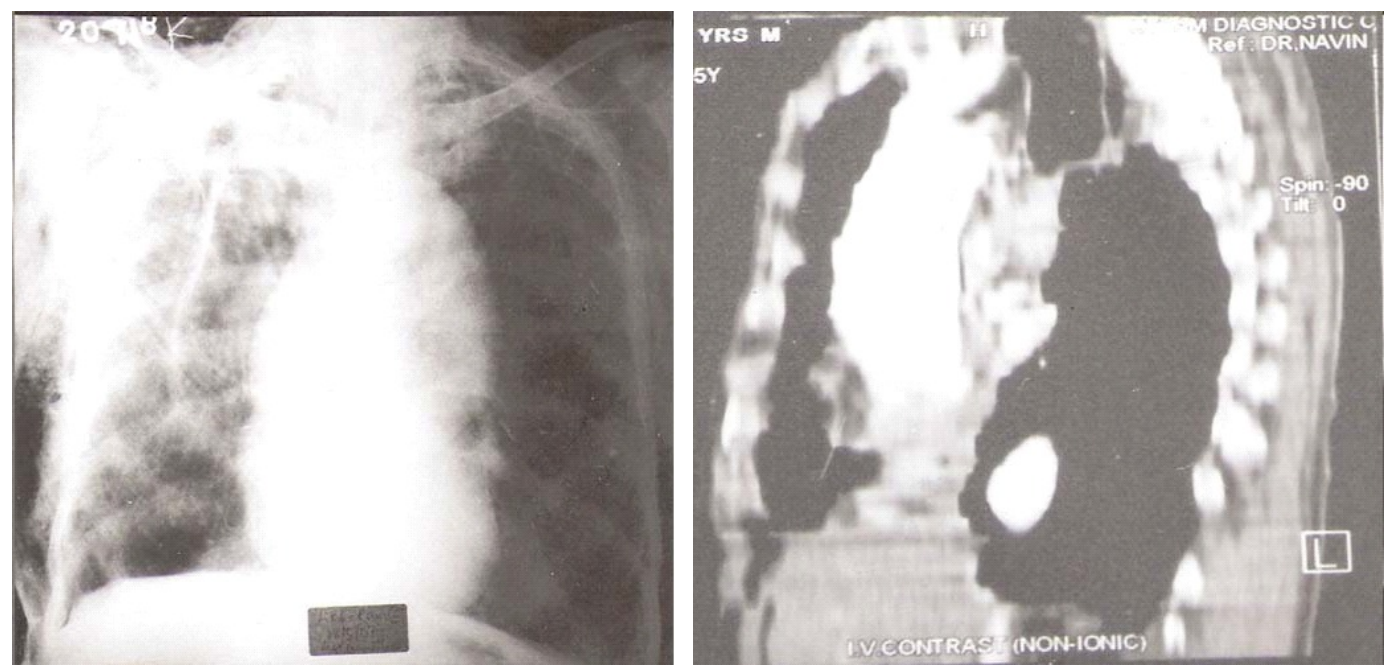

Fig. 3

Chest skiagram (PA View) and CT chest show large AA, left pneumothorax and right extensive fibrocavitatory disease 




Fig. 4

Contrast enhanced CT chest showing subcutaneous \& mediastinal emphysema, multiple bilateral bullae, right fibrocavitatory lesion, collapse left lung, centrilobular and paraseptal emphysema, aneurismal aortic dilatation in ascending, arch and descending aorta and bilateral renal cortical cysts.

In view of extensive mediastinal emphysema, intercostal drainage tube was repositioned in left thoracic cavity and connected to the under water seal. Four drug ATT regime (rifampicin, isoniazid, pyrazinamide \& ethambutol) along with tazobactum and piperacillin, ramipril (ACE Inhibitor) was started.Symptomatic and supportive treatment was also instituted. Patient improved on above treatment. Subcutaneous emphysema regressed considerably within a fortnight. There onwards patient showed remarkable improvement. (Fig. 2)

\section{Discussion}

Our patient was a chronic smoker with a smoking index of 500 . He obviously was a candidate for $\mathrm{COPD}^{3}$, hypertension, aortic aneurysm \& CAD. ${ }^{1-4}$ Interestingly he had asymptomatic large aortic aneurysm. This was no solace for us in view of inherent danger of its dissection and rupture. The reason behind smokers developing aortic aneurysm as smoking induces 5lipoxygenase expression activates 5lipoxygenase in aortic tissue which is a Key enzyme in leukotriene biosynthesis ${ }^{5}$. Further smoking promotes the formation of aneurysm through plasma macrophage inflammatory protein-1a \& 2 chemokine dependent inflammatory circuits involving both myeloid and endothelial cells. With advancing age atherosclerotic inflammation of aortic wall leads to increased propensity for penetrating aortic ulcer/dissection ${ }^{1}$. Interestingly there is significantly higher incidence of renal cysts in patients with aortic arch aneurysm (AAA) compared to patients without $\mathrm{AAA}^{4}$ as was the case in our patient.

As regards management of such cases with aortic aneurysm and hypertension it has been found that institution of ACE inhibitors decrease risk of aortic rupture by $18 \%{ }^{2}$ Patients at high risk for rupture including those over age 75 years \& those with 
hypertension seem to benefit with ACE inhibitors. What helps to these persons who have large aortic aneurysm is to stop smoking at once. ${ }^{2}$

Besides silent large $A A$, our patient had spontaneous left pneumothorax which subsequently complicated into mediastinal emphysema causing great concern. Fortunately it responded to conservative therapy. Over and above our case also had active pulmonary tuberculosis which responded to four drug ATT regimen. Presence of active tuberculosis added another twist to the etiological diagnosis for large AA. In a previous report from this country multiple saccular aneurysm of the aorta has been reported to be associated with tuberculosis ${ }^{6}$.however in our case the advanced age of patient, chronic heavy smoking, accelerated hypertension and atherosclerotic calcification in aorta favoured accelerated atherosclerosis to be the cause of large aortic aneurysm. Here again smoking was the main culprit. This case amply highlights the role of chronic smoking in the etiopathogenesis of aortic aneurysm, hypertension, renal cyst, tuberculosis and tell tale lesion of chronic obstructive pulmonary disease.

\section{References}

1. Daniel R. Wong, Walter C. Willett and Eric B. Rimm. Smoking, hypertension, alcohol consumption, and risk of abdominal aortic aneurysm in men,American journal of epidemiology 2007; 165: 838-845.

2. Diehm N, Baumgartner I. ACE inhibitor use may help prevent aortic aneurysm rupture. Lancet 2006; 368: 622-623, 659665.

3. Spencer C, Jamrozik K, Kelly S, Bremner P, Norman $P$. Is there an association between chronic lung disease and abdominal aortic aneurysm expansion? ANZ J Surg 2003; 73: 787-9.

4. Arezou Y,Christian de V,Rodney A W,Grant sarkisyan Increased Incidence of renal cysts in patients with abdominal aortic aneurysms: A common pathogenesis? Eur Respir J 2001; 18:748-752.

5. Hisato T, Takuya U. Smoking promotes pathogenesis of aortic aneurysm through the 5-lipoxygenase pathway. Med Hypotheses 2005; 64: 1117-1119.

6. Mally A., D'souza C, Dwivedi S, Shatapati P, Pulmonary tuberculosis associated with multiple saccular aneurysms of the aorta. Angiology 1990; 41: 333-336 